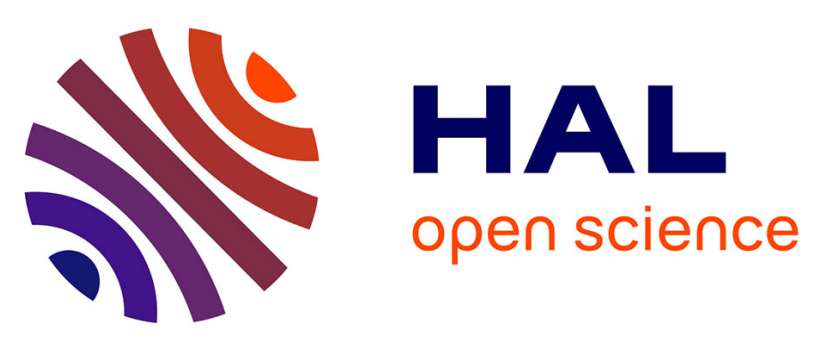

\title{
BL.Frailty: Towards an ICT-Based Platform for Frailty Assessment at Home
}

Elyes Lamine, Katarzyna Borgiel, Hervé Pingaud, Marie-Noelle Cufi, Christophe Bortolaso, Mustapha Derras

\section{To cite this version:}

Elyes Lamine, Katarzyna Borgiel, Hervé Pingaud, Marie-Noelle Cufi, Christophe Bortolaso, et al.. BL.Frailty: Towards an ICT-Based Platform for Frailty Assessment at Home. AICCSA 2019 - 16th International Conference on Computer Systems and Applications, Nov 2019, Abu Dhabi, United Arab Emirates. pp.1-6, 10.1109/AICCSA47632.2019.9035322 . hal-02525967

\section{HAL Id: hal-02525967 https://imt-mines-albi.hal.science/hal-02525967}

Submitted on 9 Feb 2021

HAL is a multi-disciplinary open access archive for the deposit and dissemination of scientific research documents, whether they are published or not. The documents may come from teaching and research institutions in France or abroad, or from public or private research centers.
L'archive ouverte pluridisciplinaire HAL, est destinée au dépôt et à la diffusion de documents scientifiques de niveau recherche, publiés ou non, émanant des établissements d'enseignement et de recherche français ou étrangers, des laboratoires publics ou privés. 


\section{BL.Frailty: Towards an ICT-Based Platform for Frailty Assessment at Home}

\author{
Elyes LAMINE \\ ISIS Engineering School / CGI \\ INUC / IMT Mines Albi-Carmaux \\ Castres / Albi, France \\ elyes.lamine@univ-jfc.fr \\ Marie-Noëlle CUFI \\ Medical Division \\ CHIC Castres-Mazamet \\ Castres, France \\ marie-noelle.cufi@chic-cm.fr
}

\author{
Katarzyna BORGIEL \\ CHL - ISIS Engineering School \\ INU Champollion (INUC) \\ Castres, France \\ katarzyna.borgiel@univ-jfc.fr \\ Christophe BORTOLASO \\ Research and Innovation Division \\ Berger-Levrault \\ Labège, France \\ christophe.bortolaso@berger-levrault.fr
}

\author{
Hervé PINGAUD \\ CHL - ISIS Engineering School / LGC \\ INUC / University of Toulouse \\ Castres / Toulouse, France \\ herve.pingaud@univ-jfc.fr \\ Mustapha DERRAS \\ Research and Innovation Division \\ Berger-Levrault \\ Labège, France \\ mustapha.derras@berger-levrault.fr
}

\begin{abstract}
Frailty is a clinical syndrome relative to ageing, and characterizes an intermediate state between robust health and the loss of autonomy. The preservation of one's capacities or abilities falls within a dependency prevention approach, based on a thorough understanding of one's medical and social situation, and his/her environment of life. This understanding is usually gained through an extensive collection of data, using standardized assessment surveys. The obtained data is next analyzed in order to provide personalized recommendations relative to the ways of living. In this paper, we describe the concept, architecture and use of an ICT platform which aims to support professionals and patients in the assessment of frailty at home. Our prototype consists of a knowledge-based system relative to assessment surveys which is connected to a smartphone application and IoT devices.
\end{abstract}

Keywords—ageing, frailty management, ICT-based platform, knowledge management, smartphone application, IoT device.

\section{INTRODUCTION}

In the context of the demographic transition towards older population, experiencing the last years of life in good health is a perspective that brings many positive implications for the elderly and for the society [1]. However, healthy and active aging does not only mean to be free from chronic diseases, but also to be away from declines in physical, cognitive or psychosocial capacities, which define the functional ability in the older age that enables well-being.

Disability and loss of independence can be triggered by illness and/or accidents, or be a consequence of decrease in physiological reserves [2]. In clinical terms, the age-related decline in physiological systems is defined as the syndrome of frailty [3]. Unlike disability, frailty is reversible or can be minimized by personalized interventions [4], aiming to preserve one's capacities. Thus, frailty assessment and personalized interventions form the basis of dependency prevention. The preventive approach aims to reduce burdens posed by disabilities not only on the elderly, but also on the healthcare system, including high healthcare costs.

In the French health system, frailty management can be seen as a decision making process. It can be described through a three stages model including: (1) frailty screening, (2) frailty evaluation and the definition of a personalized intervention plan, and (3) frailty follow-up. Hence, the aim of this management process is to prevent and/or to delay disability and dependence, and thus to improve the quality of life of older people. It aims also at reducing both the number and the length of hospitalization and institutionalization [5].

Despite the valuable potential benefits stemming from the frailty management process, its effective implementation in primary care is quite recent and still faces many challenges, particularly in terms of sustainable strategy and effective tools. This issue represents the motivations of our work.

In this paper, we describe an ongoing research project which aims the development of new assessment support for frailty management. We first provide, in section II, a brief presentation of the medical and organizational background, and we introduce main challenges related to frailty assessment. Then (section III) we describe our prototype of a ICT platform for frailty assessment. The paper ends with a discussion (section IV) and a conclusion (section V).

\section{BACKGROUND}

To understand our research motivations and the challenges to address in order to design an ICT system for frailty assessment, we must previously capture basic medical knowledge about what are the artefacts relevant to the loss of autonomy in older age, and how they can be attained in a running process of care.

\section{A. Frailty}

Frailty refers traditionally to the health status of an elderly person. It is defined as a multidimensional clinical syndrome, characterized by loss of reserves (i.e. energy, physical ability, cognition, health) and diminished resistance to stressors, causing vulnerability to adverse health outcomes $[6,7,8]$. Adverse health outcomes usually associated with frailty include: morbidity, disability, inappropriate healthcare use, institutionalization, poor quality of life, and even death [9].

There exist two main conceptualizations used to depict and to assess frailty [10]: the phenotype model [6] and the cumulative deficit model [7].

The frailty phenotype is defined across five criteriasymptoms: (1) unintentional weight loss, (2) poor handgrip strength, (3) self-reported exhaustion, (4) slow gait speed, and (5) low physical activity level. The result allows to classify an individual as one of three categories: "robust", "pre-frail" or "frail", by an ordinal value. Fried's model is related directly with the physical ability dimension.

The second model, the cumulative deficit model, quantifies a Frailty Index that is obtained by the count of clinical deficits (i.e. diseases, abilities, physical and 
neurological signs). The original list of deficits contains 70 items. The evaluation result is the ratio of health deficits present for a given individual to the total of deficits considered for this individual. Thus, frailty evaluated by Frailty Index is a continuous variable. This model covers several dimensions of frailty, including physical, cognitive, and psycho-social.

The use of these models, and associated tools, can be seen as complementary [10]: frailty phenotype was developed in order to identify frail people, while Frailty Index was created with the idea to characterize their situation in detail. Identification and characterization form the two first steps within the dependency prevention approach.

\section{B. Frailty management process}

Discriminated by the phenotype of frailty, the older population can be categorized into three sub-groups: "robust", "frail" and "disabled" elderly people [4]. Dependency prevention can be applied within each of them. In France, the master process of frailty management can be described through a three stages model (Fig. 1) including: (1) screening (or detection), (2) evaluation and personalized intervention plan (PIP) definition, and (3) follow-up [4, 11, 12].

Stage 1: Screening consists of the assessment of target individuals (i.e. 65 years old or more, without physical disability and without acute clinical disease) in order to identify pre-frail and frail elderly among the general elderly population. Frailty screening relies mostly on easy and quick assessment tools, often in the form of questionnaires, like Gérontopôle Frailty Screening Tool (GFST) [11] or Frail NonDisabled (FIND) [13]. In case of GFST, screening is done by a GP, in case of FIND - by the elderly person himself/herself.

Stage 2: Once the person is identified as pre-frail or frail, he/she can benefit from a multidisciplinary clinical assessment of different dimensions of his/her health, situation, lifestyle, and life environment. The evaluation, usually in form of a comprehensive geriatric assessment (CGA) enables the refinement of the initial diagnosis and the definition of a set of personalized prevention activities, according to the need of support. Elderly person's preferences, and the availability of resources for the realization of preventive activities, are equally considered during the formulation of recommendations. Table I resumes surveys frequently used [14], frailty dimensions covered by each survey, and the number of items (data) collected. Additional evaluations may also be proposed if necessary. The preventive interventions are behavioral and therapeutic suggestions to correct specific disability risk factors (e.g. dietary intake recommendations, physical exercises, etc.).

Stage 3: The third and last stage of frailty management process aims to follow-up the person's frailty evolution, his/her compliance to the PIP, and the evaluation of the efficiency of the proposed recommendations. At different times after the first evaluation (Stage 2), the elderly person is contacted (i.e. phone call or medical consultation) to evaluate his/her degree of engagement in new lifestyle habits and his/her situation. In the case of deterioration or maladjustment, the PIP is updated (loop from stage 3 to 2).

TABLE I. FRALTY EVALUATION SURVEYS.

\begin{tabular}{|c|c|c|c|}
\hline Survey name & $\begin{array}{l}\text { Survey } \\
\text { abbrev. }\end{array}$ & $\begin{array}{l}\text { Frailty } \\
\text { dimens. }\end{array}$ & $\begin{array}{l}\text { Nb } \\
\text { items } \\
\text { (score) }\end{array}$ \\
\hline 1. Frailty criteria (Fried) & - & Physical & $5(5)$ \\
\hline 2. Mini Mental State Examination & MMSE & Cognition & $30(30)$ \\
\hline 3. Clinical Dementia Rating & CDR & Cognition & $6(3)$ \\
\hline 4. Memory Impairment Screen & MIS & Cognition & $4(8)$ \\
\hline 5. Memory Impairment Screen - Delayed & MIS-D & Cognition & $4(8)$ \\
\hline 6. Activities of Daily Living & ADL & Physical & $6(6)$ \\
\hline 7. Instrumental Activities of Daily Living & IADL & Physical & $8(8)$ \\
\hline 8. Short Physical Performance Battery & SPPB & Physical & $3(12)$ \\
\hline 9. Gait speed & - & Physical & $1(\mathrm{n} / \mathrm{a})$ \\
\hline 10. Wrist strength & - & Physical & $1(\mathrm{n} / \mathrm{a})$ \\
\hline 11. Mini Nutritional Assessment & MNA & Nutrition & $18(30)$ \\
\hline 12. Vitamin D concentration & - & Nutrition & $1(\mathrm{n} / \mathrm{a})$ \\
\hline 13. Geriatric Depression Scale & GDS & $\begin{array}{l}\text { Psycho- } \\
\text { social }\end{array}$ & $15(15)$ \\
\hline 14. Distance vision & - & Sensory & $1(\mathrm{n} / \mathrm{a})$ \\
\hline 15. Near vision & - & Sensory & $1(\mathrm{n} / \mathrm{a})$ \\
\hline 16. Amsler grid & - & Sensory & $1(\mathrm{n} / \mathrm{a})$ \\
\hline $\begin{array}{l}\text { 17. Hearing Handicap Inventory for the } \\
\text { Elderly - Screening }\end{array}$ & HHIE-S & Sensory & $10(40)$ \\
\hline 18. Urinary incontinence & - & Other & $6(6)$ \\
\hline 19. Oral Health Assessment Tool & OHAT & Other & $8(16)$ \\
\hline SUM number of items (score) & & & 123 \\
\hline
\end{tabular}

In France, the general practitioner plays an important role in all three stages of such a frailty management process, being the main process owner on the medical side.

\section{Frailty management challenges}

Although the theoretical concept of frailty is agreed upon, its practical screening and evaluation still present some limitations due to the existence of a multitude of frailty assessment surveys $[8,15,16,17,18]$. One could point the choice of the right set of surveys as the main challenge within the frailty management tools. The choice of the data to use to assess the person's situation is clearly of utmost importance, but the differences in their use between professionals coming from different institutions or countries is more a cultural issue.

Given the number of evaluation surveys (e.g.19 in the example in Table I), the evaluation and the definition of personalized intervention plan are quite time consuming. Moreover, evaluation is often done in a hospital, by a group of healthcare professionals (i.e. geriatrician, nurse, neuropsychologist, physical therapist, dietician), who must be

Elderly evolution process

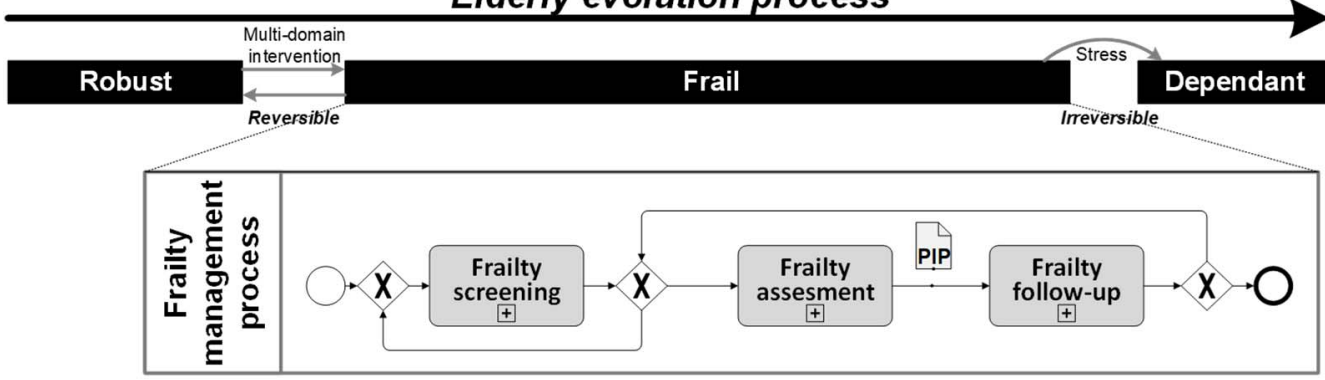

Fig. 1. The process of elderly evolution and the process of frailty management. 
seen as limited resources. Thus, the number of elderly people who actually undergo screening and evaluation remains low compared to their actual number in a given territory.

In the light of these limitations, there is an urgent need to implement home-based frailty management, relying on home care services, and using digital solutions for data collection. It is noteworthy that each stage of frailty management process faces specific challenge relative to data collection. During screening (Stage 1), the challenge is to get in touch with and collect the data form the whole population of target individuals (i.e. people of 65 years or more). During evaluation and PIP definition (Stage 2), the challenge is to collect important amounts of data in the most efficient way, both for the evaluated person, and for the evaluators (professionals). During follow-up (Stage 3), the challenge is to collect personalized sets of data, over long periods of time (months to years) and with high frequency (several times during a year), in order to detect changes as they occur.

To deal with these challenges, many research projects aim the design and/or the evaluation of technological artefacts for automated collection of frailty-related indicators, with the idea to augment and/or to replace traditional paper support $[19,20]$. Among them, we can name individual wearable artefacts for automated assessment of activity patterns by objective uniaxial accelerometer [21], or of the muscular strength during stair climbing by using 3D-accelerometers and 3D-gyroscopes [22]. As the extension of person-embedded sensors systems, are projects aiming environment-embedded systems composed of multiple sensors. For example, Indoor Proximity Systems are experimented in order to monitor indoor patient activity [23]. In both cases, the use of sensors enables more frequent data collection and objective measurements [22]. There are other research projects that aim the design and/or the evaluation of technologies supporting the user entry of frailty-related indicators, including self-assessment by the elderly (FrailSafe by porto4ageing). Finally, there exist also projects that support mixed data collection methods, including both manual and automatic. For example, data can be collected from sensors and from accelerometer sensors in a mobile phone and from the patient record [24].

Given the need to support home-based frailty management, our goal is to provide an ICT platform, named BL.Frailty, dedicated for frailty assessment (screening, evaluation and follow-up) at home. In comparison with related work, our motivation is to provide an adaptable data collection platform through a mix of automatic, semi-automatic and manual data entry solutions. The automatic data entry is to be based not on the creation of new devices, but on the integration of already existing solutions. The semi-automatic data collection will be based on the study of assessment surveys. If the ultimate goal is to assist frailty assessment process by automating some assessment activities, the automation is not always feasible nor desirable, and the manual-automatic data collection will also be made possible. Thus, for each data traditionally used to assess frailty, we aim to provide, where possible, several alternative data collection methods. This way, we want to provide the users (the elderly and professionals) with the possibility of creating, for each assessment case, a personalized data collection system, depending on their needs, preferences, constraints, etc.

\section{BL.FRAILTY PLATFORM OVERVIEW}

In order to study the opportunities for automation of assessment activities within the frailty management process, we have first analyzed the content of different assessment surveys used by a local hospital center, offering frailty evaluation service within one-day hospitalization. The analysis of data covered by each survey rapidly showed that there exist similarities between them, more precisely in the topics and the variables that they cover. In order to identify the similarities in a rigorous manner, we carried out a semantic mapping of the content of 29 surveys (Table II).

\section{TABLE II. EXTRACT OF THE KNOWLADGE DATABASE OF VARIABLES COVERED BY ASSESSMENT SURVEYS.}

\begin{tabular}{|l|c|c|c|c|}
\hline \multicolumn{5}{|c|}{ Extract of assessment surveys } \\
Extract of variables & FIND & GFST & ADL & MNA \\
\hline Difficulties in walking, moving around & + & + & + & + \\
\hline Difficulties in stair climbing & + & & & \\
\hline Slow gait speed & & + & & \\
\hline Weight loss & + & + & & \\
\hline Fatigue / effort & + & + & & \\
\hline Physical activity level & + & & & \\
\hline Living alone & & + & & \\
\hline Memory complaints & & + & & \\
\hline Autonomy in feeding & & & + & + \\
\hline
\end{tabular}

Once we have defined the variable assessed by each question and by each possible answer for a question, we specified two types of semantic links (Fig. 2). The first type, called "similar to", expresses a semantic equivalence relationship between two concepts. This is a two-way relationship. The second type, called "relating to", expresses a closely related relationship between two concepts, and is a unidirectional relationship.

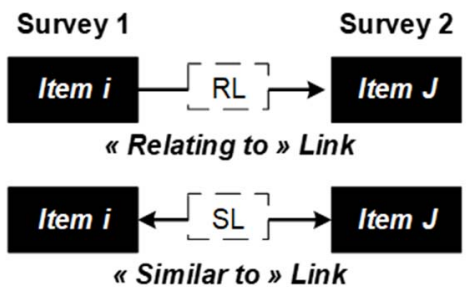

Fig. 2. Two types of links between assessment questions.

We have used these two semantic link types to map question items addressing the same variable, with the same meaning, among different assessment surveys.

For example, the MNA survey and the ADL survey both include the assessment of the ability of an elderly person to feed himself/herself and his/her ability to move around. In the first case, the MNA question item is "Mode of feeding", and it has three possible answers. The ADL question item is "Feeding", with three possible answers in the French version. The result of mapping of possible answers for these two questions for these two surveys are four one-way semantic links (Fig. 3, at the top).

MNA and ADL both serve as well to evaluate the ability of the elderly person to move. The ADL question is "Transferring", and the MNA question is "Mobility". However, the meaning of the evaluation items is not exactly the same in these two surveys, thus there is only one mapping possible (Fig. 3, at the bottom). In this case, the identified semantic link is two-way.

\section{A. BL.Frailty platform concept}

The identification of links between assessment surveys was at the origin of the concept of the future platform to 


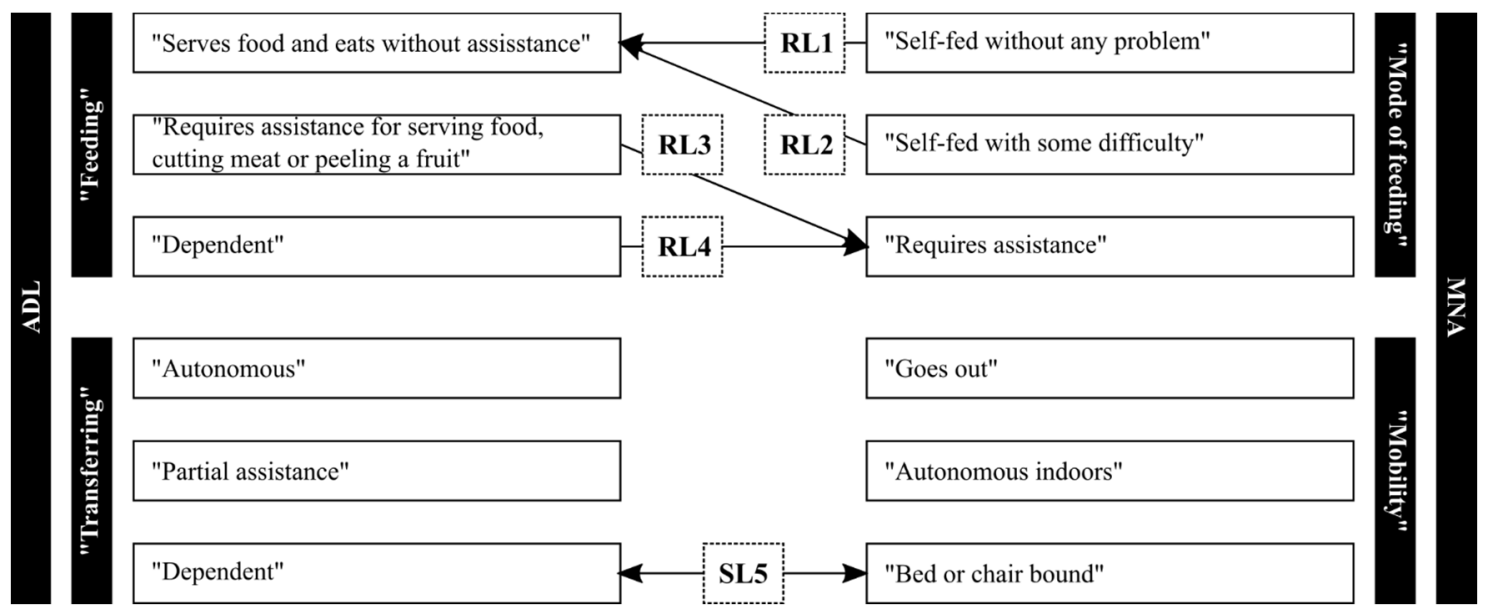

Fig. 3. Semantic links "relating to" (RL) and "similar to" (SL) resulting from mapping between MNA and ADL surveys.

design. Indeed, by providing such a knowledge base of the surveys and their semantic connections, the system could assist the user in the evaluation process by suggesting to complete at the same time all of the questions addressing the same variable in the same manner. Thus, the first functional requirement for the platform was to support frailty assessment by reusing data collected and entered by the evaluator (user).

As the possible propagation should always remain under the control of the user, the platform was also to be designed to support frailty assessment by allowing the manual entry and the storage of all assessment data. The support role of the technological media is here more traditional, and relies in the capacity to aid the calculation of evaluation scores, to access historical data, and to represent it textually and graphically.

Finally, with the idea of data collection without the presence of any professional, the system was to support frailty assessment by automatic data collection from sensors. Here, our goal was not to develop new ways of collecting data, but to reuse already existing technologies.

Thus, the future platform should give both manual, semiautomatic and automatic support for frailty data entry. In order to experiment the validity of the concept, we have worked on a first prototype of the ICT platform.

\section{B. Surveys and functionalities}

The prototype implements at this stage two screening and nine evaluation surveys (stage 1 and stage 2). Screening surveys are (1) GFST and (2) FIND. Evaluation surveys cover: (1) ADL, (2) IADL, (3) SPPB, (4) Tinetti, (5) MNA, (6) MMSE, (7) GDS, (8) Doloplus and (9) Algoplus. Moreover, the prototype covers weight and BMI. measures

The prototype of the ICT platform encompasses eight main functionalities (digital services): (1) survey and measures management, (2) knowledge base management, (3) user management, (4) patient management, (5) administrative patient data entry, storage and display, (6) medical patient data entry, storage and display, (7) calculation of assessment survey scores, and (8) creation and displays of graphs of assessment survey results and measures.

\section{Smartphone application}

The smartphone application is the principal part of the platform. It has three main components: (1) connection page, (2) patient list, and (3) patient assessment record. Patient assessment record includes: (3.1) patient administrative data,
(3.2) assessment surveys, (3.3) assessment measures, and (3.4) assessment charts (Fig. 4). The connection page guarantees patient data security and is enabled by a username and a password. Once the user is connected, the application displays the list of patients associated with the user. By choosing a given patient name, the user enters the patient assessment record. The main page of the assessment record is composed of patient administrative data and of five navigation icons. The icons enable to access assessment surveys, assessment questions, measures, assessment history, and to include data collected by sensors (Fig. 4-1).

The list of assessment surveys shows not only the names of different surveys, but also - if one or many of surveys have already been used for the patient - the score obtained during the last evaluation, the day of the last evaluation and the name of the evaluator (the user; Fig. 4-2). After opening a given survey, the user visualizes different survey questions and question items (possible answers). Also, if the survey has already been used in the past, for each question the user visualizes question items used during the last evaluation (Fig. 4-3). Thus, a new evaluation is always carried out in reference to the previous one, and the evaluation data is entered as modifications of question items.

Similarly, the list of measures shows measure names and, if applicable, the last obtained value, the day and the person who collected and entered the data.

Assessment history enables to consult all past evaluations (score, date, hour and username) in chronological order and under two representations: textual and graphical (Fig. 4-4).

In line with the concept, the smartphone application includes two forms of data collection: manual and semiautomatic propagation of evaluation results. Indeed, the presence of semantic links enables to assist the user during the assessment process. For example, while completing the MNA, if the user choses "Self-fed without any problem", the interface offers him the possibility two answer in the same time the ADL question by entering "Serves food and eats without assistance" (Fig. 4-5).

\section{Sensor}

The sensor that we have decided to experiment in the first step, in order to enable the automatic collection of data, is a weight \& BMI (Body Mass Index) Wi-Fi scale, the scale "Body" by Nokia Withings. It enables to collect automatically the weight of the person, and his/her BMI. 

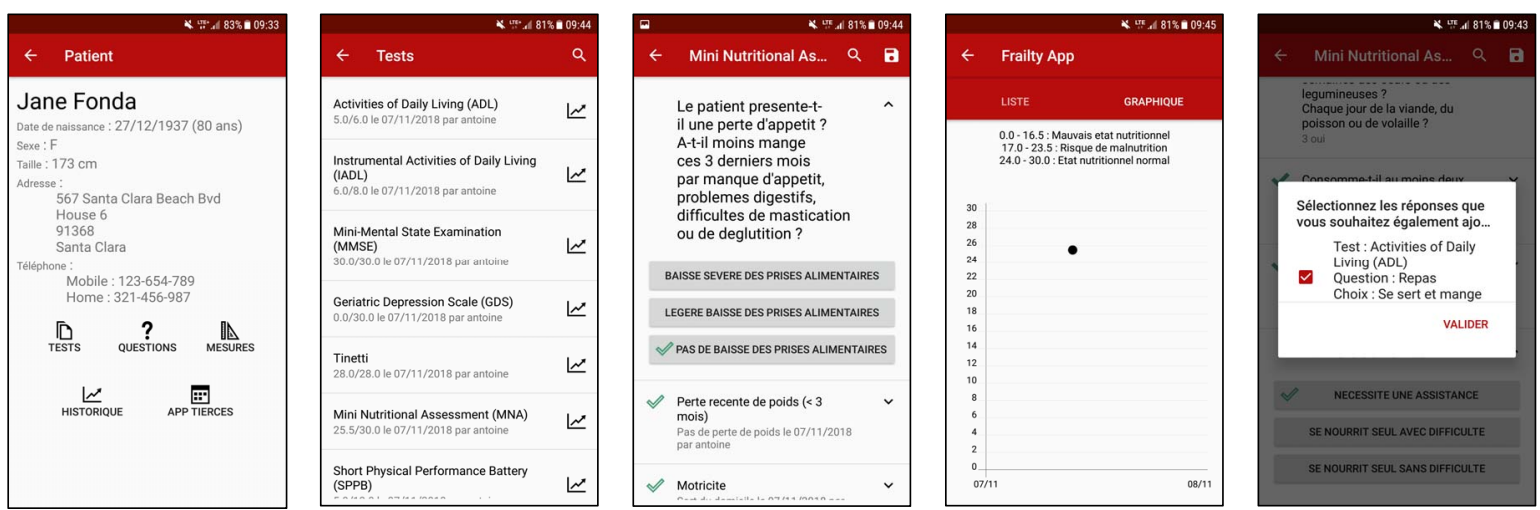

Fig. 5. Smartphone application (from the left): (4-1) patient assessment record, (4-2) list of tests, (4-3) MNA test entry (on the left); (4-4) automatic proposition of answer for a question of ADL test, while doing MNA test, (4-5) MNA test score graph.

\section{E. Architecture}

The architecture of BL.Frailty consists of: (1) a server, (2) an administration web interface, (3) a smartphone application, and (4) a sensor (Fig. 5).

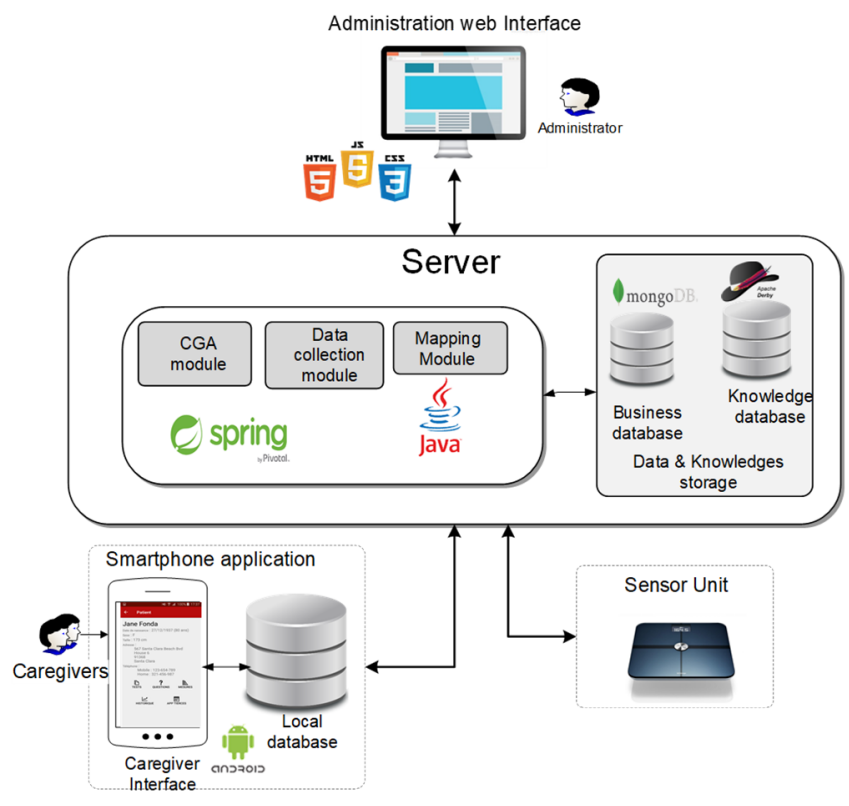

Fig. 4. Architecture of the system.

The server was developed in Java and communicates both with the interface, the mobile application, and the sensor system, through web services. The database on the server is divided in two parts. The first part, a SQL Database 1 (MySQL), contains static data relative to surveys (i.e. survey names, survey questions, possible answers, knowledge base of semantic links), to measures (measure list), to users (user list and rights) and to patients (patient list and their administrative data). The second part, a NoSQL database 2 (MongoDB), contains dynamic data relative to results of patient evaluations (answers given during an evaluation, values of measures).

The administration web interface was developed with standard web technologies (HTML, CSS and JavaScript). The interface enables to manage (addition, modification, suppression) the list of surveys and measures, the knowledge database, the list of users and the list of patients.

The mobile application was developed in Android. It operates thanks to a user interface and a local SQLite database, which communicates with the server databases. The access to the user interface requires a user account. Despite the presence of a local database, the use of the mobile application requires constant access to the server in order to guarantee the security of patient data, through constant control of user rights.

The "Body" scale holds its proper database, and offers a smartphone application enabling to monitor collected data. The interface between the server and the scale is done through the communication with the scale's API. At this stage of the project, the user has to manually request the update of data, which are next registered in the BL.Frailty database.

\section{DISCUSSION}

In order to address the definition of the concept and the design of a new technological solution for frailty assessment, we have adopted the Living Lab design methodology based on a partnership between local actors: (1) a software company, (2) an engineering school and its living lab, specialized in academic and applied research in e-health, and (3) with a local hospital center specialized in frailty management. This approach allowed us to integrate future users, healthcare professionals in our case, in the development process from the first stage of the project. Its output is the prototype of the BL.Fraility platform. This first version includes manual data entry; automatic data collection by electronic body weight scale "Body" from Nokia Withings; and semi-automatic propagation of evaluation results, based on the knowledge base of semantic links within the nine evaluation surveys.

Overall, there is only one survey, IADL, for which no semantic link with other survey has been identified. For the eight surveys, we identified 32 semantic links. Among these, eight links are "similar to" (two-way) links (Table III). The remaining 24 links are "relating to" (one-way) links (Table IV).

TABLE III. “SIMILAR TO” LINKS BETWEEN SURVEYS.

\begin{tabular}{|l|c|l|}
\hline Between survey & Number of links & and survey \\
\hline ADL & 1 & MNA \\
\hline Doloplus & 2 & ADL \\
\hline Doloplus & 1 & GDS \\
\hline Doloplus & 4 & Algoplus \\
\hline
\end{tabular}

TABLE IV. “REALTING TO" (LINKS BETWEEN SURVEYS.

\begin{tabular}{|l|c|l|}
\hline From survey & Number of links & Towards survey \\
\hline ADL & 2 & MNA \\
\hline MNA & 2 & ADL \\
\hline Tinetti & 2 & SPPB \\
\hline Doloplus & 4 & ADL \\
\hline Doloplus & 2 & GDS \\
\hline Doloplus & 12 & Algoplus \\
\hline
\end{tabular}


Overall, the number of links identified between surveys was the highest for surveys aiming the assessment of the same domain (i.e. pain assessment with Doloplus and Algoplus). In the case of complementary surveys, the links were also present but less numerous. These first results show that the knowledge database of semantic links can provide support for the frailty management process, however this kind of data entry assistance will apply only to a subset of assessment variables. The effective support needs to be further studied, both based on the integration of other surveys (i.e. Table I) and on its application within real-life frailty management practice.

Our proposal to support frailty assessment at home, developed in this paper, is to provide an adaptable data collection platform, that would enable to create personalized frailty assessment systems based on the integration of a mobile application, of a knowledge database and of sensors. We identify several research directions for future work. First, within the idea of alternative data collection methods, we plan to study the value of these different methods, which can possibility be different depending on the assessment variable in question. Next, as the platform relies on the integration of sensors already existing on the market, and as usually there exist several different sensors for collecting a given data (i.e. weight), we must consider the value of usage of these alternative sensors in order to promote the acceptability of the platform and its components by the end users. Also, as the flexibility of the platform could also come from the personalization of manual data entry, we plan also to study the value of data entry by non-healthcare professionals, including elderly people themselves. Finally, we plan to evaluate the usability of the mobile application, with professionals and elderly people, both in laboratory and natural settings.

\section{CONCLUSION}

In order to address data collection challenges within the dependency prevention approach, we have developed a concept and a prototype of an ICT platform for frailty assessment. The concept of the system is based on a mix of manual, semi-automatic (knowledge base) and automatic (sensors) data collection techniques. The prototype of the system implements successfully these three techniques and thus forms a valid proof of concept.

\section{ACKNOWLEDGMENTS}

The authors would like to thank A. Lefort (CHIC-CM) for her precious insights on the frailty management process running and challenges. A. Lapeyre (ISIS) has realized the research and development work of the first prototype, which was subsequently continued by T. Frassati and S. Tournou (ISIS). C. Picard and V. Morel (Berger-Levrault) have both contributed to the creation and to the execution of this project.

\section{REFERENCES}

[1] World report on ageing and health. Geneva: World Health Organization, 2015..

[2] J.-M. Robine and S. Andrieu, "Introducing frailty and the frailty process within the disablement models,"in The White Book on Frailty, B. Vellas, Ed. International Association of Gerontology and Geriatrics, 2016, pp. 13-14.

[3] A. Clegg, J. Young, S. Iliffe et al. , "Frailty in elderly people," Lancet, vol. 381, pp. 752-762, Feb. 2013.

[4] J. Subra, S. Gillette-Guyonnet, M. Cesari et al., "The integration of frailty into clinical practice: preliminary results from the Gérontopôle," in The White Book on Frailty, B. Vellas, Ed. IAGG, 2016, pp. 132139.

[5] M. Iglesia-Gomez, "Action group on prevention of frailty of the European Innovation Partnership (EIP) on active and healthy ageing," in The White Book on Frailty, B. Vellas, Ed. IAGG, 2016, pp. 10-12.

[6] L. P. Fried, C. M. Tangen, J. Walstonet al., "Frailty in Older Adults: Evidence for a Phenotype," Journal of Gerontology: Medical Sciences, vol. 56A, no. 3, pp.M146-M156, Mar. 2001.

[7] K. Rockwood, X. Song, C. MacKnightet al., "A global clinical measure of fitness and frailty in elderly people," Canadian Medical Association Journal, vol. 173, no. 5, pp. 489-495, Aug. 2005.

[8] D.B. Hogan, "Models, Definitions, and Criteria for Failty," in Conn's Hadbook of Models for Human Aging, 2nd ed., J. L. Ram and P. M. Conn, Eds. (Academic Press, 2018, pp. 35-44.

[9] E. Marzetti, R. Calvani, F. Landiet al., "Innovative Medicines Initiative: The SPRINTT Project," Journal of Frailty and Aging, vol. 4, no. 4, pp. 207-208, Dec. 2015.

[10] M. Cesari, G. Gambassi, G. A. van Kan, and B. Vellas , "The frailty phenotype and the frailty index: different instruments for different purposes," Age and Ageing, vol. 43, no. 1, pp. 10-12, Jan. 2014.

[11] B. Vellas, L. Ballardy, S. Gillette-Guyonnet et al., "Looking for frailty in community-dwelling older persons: The Gérontopôle Frailty Screening Tool (GFST)," in The White Book on Frailty, B. Vellas, Ed. IAGG, 2016, pp. 129-131.

[12] J. de Kerimel, N. Tavassoli, C. Lafont et al., "How to Manage Frail Older Adults in the Community? Proposal of a Health Promotion Program Experienced in a City of 16,638 Inhabitants in France," Journal of Frailty and Aging, vol. 7, no. 2, pp.120-126, Feb. 2018.

[13] M. Cesari, L. Demougeot, H. Boccalon et al., "A Self-Reported Screening Tool for Detecting Community-Dwelling Older Persons with Frailty Syndrome in the Absence of Mobility Disability: The FiND Questionnaire," PLoS ONE, vol. 9, no. 7, e101745, Jul. 2014.

[14] B. Vellas., "Implementing frailty screening, assessment, and sustained intervention: The experience of the Gérontopôle," in The White Book on Frailty, B. Vellas, Ed.. IAGG, 2016, pp. 120-128.

[15] N. M. de Vries, J. B. Staal, C. D. van Ravensberg et al., "Outcome instruments to measure frailty: A systematic review," Ageing Research Reviews, vol. 10, no. 1, pp. 104-114, Jan. 2011.

[16] S. A. Sternberg, A. Wershof Schwartz, S. Karunananthan et al., "The Identification of Frailty: A Systematic Literature Review," Journal of the American Geriatrics Society, vol. 59, no. 11, pp. 2129-2138, Nov. 2011.

[17] B. J. Buta, J. D. Walston, J. G. Godino et al., "Frailty assessment instruments: Systematic characterization of the uses and contexts of highly-cited instruments," Ageing Research Reviews, vol. 26, pp. 5361, Mar. 2016.

[18] B. Xie, J. L. Larson, R. Gonzalez et al., "Components and indicators of frailty measures: a literature review," The Journal of Frailty \& Aging, vol. 6, no. 2, pp. 76-82, 2017.

[19] E. Kelaiditi, "Frailty and Novel Technologies - A Step Ahead," Journal of Frailty and Ageing, vol. 4, no. 2, pp. 90-92, 2015.

[20] I. Mugueta-Aguinaga and B. Garcia-Zapirain, "Is Technology Present in Frailty? Technology a Back-up Tool for Dealing with Frailty in the Elderly: A Systematic Review,"Ageind and Disease, vol. 8, no. 2, pp. 176-195, Apr. 2017.

[21] C.S. Chan, S.E. Slaughter, C.A. Jones, A.S. Wagg, "Measuring activity performance of continuing care residents using the activpal: an exploratory study," The Journal of Frailty \& Aging, vol. 5, no. 3, pp. 158-161, 2016.

[22] S. Hellmers, T. Kromke, L. Dasenbrock, A. Heinks, J. M. Bauer, A. Hein, and S. Fudickar, "Stair Climb Power Measurements via Inertial Measurement Units. Towards an Unsupervised Assessment of Strength in Domestic Environments," presented at the Proceeding of BIOSTEC, vol. 5 : HEALTHINF, Funchal, Portugal, 2018, pp. 39-47.

[23] L. Power, L. Jackson, and S. Dunett., "Developing a Sensor based Homecare System. The Role of Bluetooth Low-Energy in Activity Monitoring," presented at the Proceeding of BIOSTEC, vol. 5 : HEALTHINF, Funchal, Portugal, 2018, pp. 598-606.

[24] J. Fontecha, R. Hervás, J. Bravo, and F. J. Navarro, "A Mobile and Ubiquitous Approach for Supporting Frailty Assessment in Elderly People," Journal of Medical Internet Research, vol. 15, no. 9, e197, Sep. 2013. 